\title{
Unexpected Molecular Flip in Solid-State Photodimerization
}

\author{
Thekku Veedu Sreevidya, ${ }^{\dagger, \S}$ Deng-Ke Cao, ${ }^{\dagger, \dagger}$ Tali Lavy, ${ }^{\dagger}$ Mark Botoshansky, ${ }^{\dagger}$ and Menahem Kaftory ${ }^{*}{ }^{\dagger}$ \\ ${ }^{\dagger}$ Schulich Faculty of Chemistry, Technion-Israel Institute of Technology, Haifa 32000, Israel
}

Supporting Information

ABSTRACT: Three cocrystals of the light-stable compound 1,1,6,6-tetraphenyl-2,4-hexadiyne-1,6-diol (I) with light-sensitive molecules 1,2-dimethyl-2(1H)-pyridinone (a), 6-methyl-2(1H)pyridinone (b) and 2-methyl-2(1H)-pyridinone (c) were exposed to UV light. It was found that the molecules undergo molecular flip perpendicular to the molecular plane (rotation of $\sim 180^{\circ}$ ). In the first two cocrystals, the light-sensitive molecules are disordered, which means that the space provided for them is larger than needed for ordered molecules. Therefore, rotation can take place. Moreover, in I-b, the flip is temperature dependent and takes

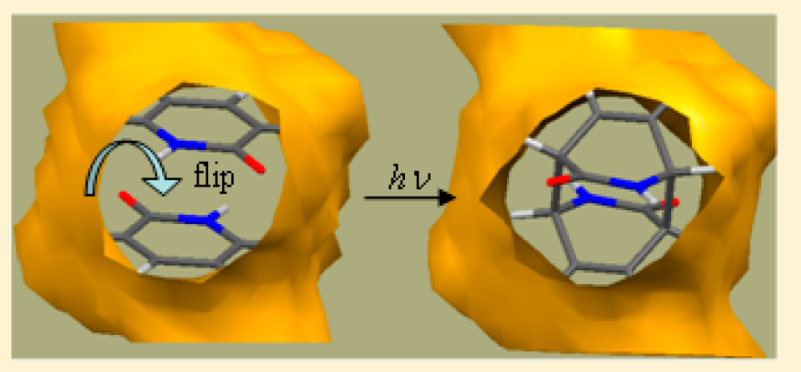
place without exposure to UV light. Crystal structures at four different temperatures enable one to estimate the activation energy of the flip to be $9.72 \mathrm{~kJ} / \mathrm{mol}$. The kinetics of the reaction of I-c was studied at room temperature and revealed a sigmoidal behavior with Avrami exponent of $n=0.95(6)$ that could be explained by the JMAK model for crystal growth. It means that the nucleation rate is constant over time and that the reaction is homogeneous with equal probability to occur in any region of the sample. This could be explained by the fact that the voids where the reaction and the flip take place are isolated from each other.

\section{INTRODUCTION}

There is a major advantage of studying solid-state photochemical reactions of systems where the transformation from the reactant to the product takes place homogenously, from single-crystal to single-crystal. In all other cases where structural information is lost, partially or completely, one has to speculate on the events during the reaction, and one can miss some of them. We have recently come across three examples in which the structural behavior of the reactant molecules could not be envisaged. We succeeded to observe the unexpected events only due to the fact that the transformation from reactants to products took place in a single-crystal to single-crystal mode. It was recently demonstrated that in cocrystals composed of lightstable host molecules and light-sensitive guest molecules, the photochemical reaction may proceed to completion with the preservation of the single-crystal integrity. ${ }^{1-9}$ Such systems enable kinetic investigation of compounds that do not undergo photochemical reactions in the solid state in their neat phase. ${ }^{10}$ It also enables the study of the effects of the environment on the reaction product. ${ }^{1,11}$

In this Article, we would like to describe molecular flip (rotation by $180^{\circ}$ ) while a $[4+4]$ photodimerization takes place in a system composed of light-stable host (I) and lightsensitive guest molecules $\mathbf{a}, \mathbf{b}$, and $\mathbf{c}$ (Scheme 1). The flip was observed to take place as a result of heat or of illumination by UV light. Each example will be described separately, and because the crystal structure of I-b, I-c was described in detail in previous publication, ${ }^{4}$ we will mention the structures briefly. The crystal structure of I-a is new; therefore, we describe it in more detail.
Scheme 1

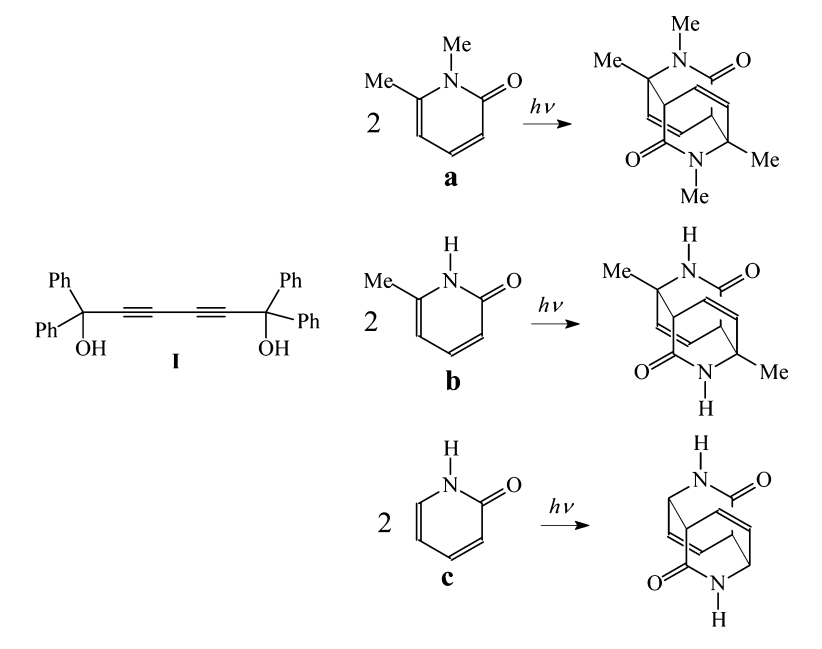

\section{EXPERIMENTAL SECTION}

Preparation of the Materials. Commercially available reagents were purchased from Aldrich and used without further purification.

1,2-Dimethyl-2(1H)-pyridinone (a). The mixture of 2-methyl-2pyridinone $(9.16 \mathrm{mmol}, 1.0 \mathrm{~g})$, potassium carbonate $(35 \mathrm{mmol}, 4.82$ g), methyl iodide $(35 \mathrm{mmol}, 2.2 \mathrm{~mL})$, and acetone $(100 \mathrm{~mL})$ was refluxed for $4 \mathrm{~h}$ in oil bath $\left(80^{\circ} \mathrm{C}\right)$. After being cooled, potassium carbonate was removed. The filtrate was evaporated, and the resulting residue was mixed with $12 \mathrm{~mL}$ of water, and then extracted with

Received: November 14, 2012

Revised: December 27, 2012

Published: January 7, 2013 
chloroform $(45 \mathrm{~mL} \times 3)$. The chloroform solutions were dried with $\mathrm{MgSO}_{4}$. After removal of chloroform, the resulting residue was distilled $\left(92{ }^{\circ} \mathrm{C}, 0.5 \mathrm{mbar}\right)$ and was crystallized from dry ether. ${ }^{12}$

6-Methyl-2(1H)-pyridinone (b) and 2-methyl-2(1H)-pyridinone (c) are commercially available.

1,1,6,6-Tetraphenyl-2,4-hexadiyne-1,6-diol (I). $\mathrm{Cu}(\mathrm{OAc})_{2}$ (5 mmol, $0.9 \mathrm{~g}$ ) was dissolved in $60 \mathrm{~mL}$ of methanol-pyridine (1:1), and then the solution of 1,1-diphenylpropyn-1-ol (purchased from Sigma-Aldrich) (10 mmol, $2.08 \mathrm{~g})$ in $60 \mathrm{~mL}$ of methanol-pyridine (purchased from Sigma-Aldrich) (1:1) was added dropwise with stirring in an oil bath $\left(63^{\circ} \mathrm{C}\right)$. After the mixture was stirred for $10 \mathrm{~h}$, it was cooled to room temperature. The solvent was evaporated, and the resulting residue was mixed with $100 \mathrm{~mL}$ of ether- $\mathrm{CS}_{2}(\mathrm{v} / \mathrm{v} 4 / 1)$ and $250 \mathrm{~mL}$ of $2 \mathrm{M} \mathrm{HCl}$. The organic phase of the mixture was separated, and the aqueous phase was further extracted with $2 \times 100 \mathrm{~mL}$ of ether $-\mathrm{CS}_{2}(\mathrm{v} / \mathrm{v} 4 / 1)$. Finally, the combined organic phase was washed with $3 \times 100 \mathrm{~mL}$ of $2 \mathrm{M} \mathrm{HCl}, 3 \times 100 \mathrm{~mL}$ of water, and $3 \times 100 \mathrm{~mL}$ of $\mathrm{NaCl}$ solution, and dried with $\mathrm{MgSO}_{4}$. After removal of solvent, the pale yellow residue was refluxed in hexane $(40 \mathrm{~mL})$ to yield powder. After recrystallization in $\mathrm{CH}_{2} \mathrm{Cl}_{2}$-hexane, the colorless needle-like crystals were collected with $89 \%$ yield. $^{13}$

Cocrystals I-a, I-b, and I-c. 1:2 equivalent proportion of the components was dissolved in a mixture of ethyl acetate and methanol. The solution was stirred at room temperature for $1 \mathrm{~h}$. The solution was evaporated in air for a few days until colorless crystals appeared.

Irradiation Setup. The irradiation system consisted of an Osram Xe short arc lamp ( $150 \mathrm{~W})$, in the irradiation of $\mathbf{I}-\mathbf{b}$, and with a LED source (UV-LED) in the range 350-390 $\mathrm{nm}$ with the maximum of $365 \mathrm{~nm}$ for the irradiation of I-a and I-c. Each single crystal was attached with grease to a thin piece of glass and mounted at a distance of about $2 \mathrm{~cm}$ in front of the focused beam on a device that revolved at $1 \mathrm{rpm}$. After each stage, diffraction intensity data were collected. Conversion percentage was determined by the occupancy factors obtained from the crystal structure refinement.

Crystal Structure Determination and Refinement. Intensity data have been collected on a KapaCCD Nonius diffractometer or on a Bruker APEX2 Duo diffractometer (Mo K $\alpha, \lambda=0.71073 \AA$ ). The following software has been used for crystal structure solution and refinement: data collection, COLLECT; ${ }^{14}$ cell refinement and data reduction, DENZO-SMN. ${ }^{15}$ The program used to solve and refine the crystal structures was SHELXS-97. ${ }^{16}$ The program used for molecular graphics was MERCURY. ${ }^{17}$

Before and after irradiation to full conversion, all non-hydrogen atoms were refined anisotropically. The positions of the hydrogen atoms were located in difference Fourier maps and refined by riding on their parent atoms. At intermediate stages of irradiation, the refinement of the crystal structure that includes the unreacted monomers of the light-sensitive molecule and the partially produced dimer was carried out in a procedure similar to that used above; however, the occupancy factor of the atoms of the unreacted molecules and the product molecules was refined. At lower and higher conversion, the atoms of the minor species were refined isotropically; at conversion closer to $50 \%$, both unreacted molecules and the product molecules were refined anisotropically.

\section{RESULTS}

3.1. 1,1,6,6-Tetraphenyl-2,4-hexadiyne-1,6-diol:2(1,6dimethyl-2(1H)-pyridone) (I-a). The molecular compound crystallizes in the monoclinic system $P 2_{1} / c$ space group. The structure may be generally described as made up of 1,6dimethyl-2 $(1 H)$-pyridone (a) molecules forming dimers through hydrogen bonds. They are also hydrogen bonded to the host molecule 1,1,6,6-tetraphenyl-2,4-hexadiyne-1,6-diol. The light-sensitive (a) molecule is disordered in equal proportion as seen in Figure 1.

Those molecules are related by inversion center to other disordered molecules. The geometric relation between each pair of potentially reacting molecules to form dimers is shown

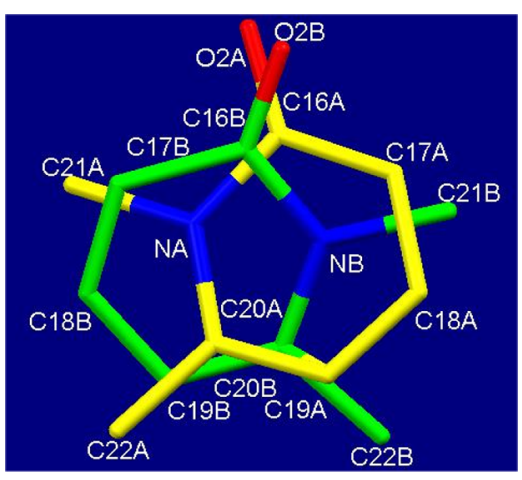

Figure 1. The disordered light-sensitive molecule in I-a.

in Figure 2. There are three possible interactions that may lead to photodimers: molecules $\mathrm{A}-\mathrm{A}, \mathrm{B}-\mathrm{B}$, and $\mathrm{A}-\mathrm{B}$. The last $(\mathrm{A}-$

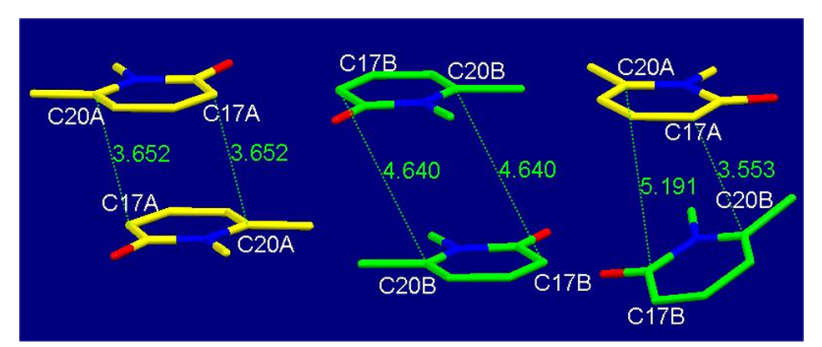

Figure 2. Relative geometry of light-sensitive molecules in I-a. A ‥A (left), $\mathrm{B} \cdots \mathrm{B}$ (middle), and $\mathrm{A} \cdots \mathrm{B}$ (right). Carbon atoms of molecule A are in yellow, and those of molecule B are in green.

B) is unlikely to be present at the same void due to the less favorable interactions. The distance between the potentially reactive atoms $\mathrm{C} 17 \mathrm{~A}$ and $\mathrm{C} 20 \mathrm{~A}$ with their counterparts related by inversion center, $\mathrm{C} 20 \mathrm{~A}^{\mathrm{i}}$ and $\mathrm{C} 17 \mathrm{~A}^{\mathrm{i}}$, is $3.652(3) \AA$ (see Figure 2) shorter than the upper limit of $4.2 \AA$, set by Schmidt. ${ }^{18}$ The equivalent distances between atoms $\mathrm{C} 17 \mathrm{~B}$ and $\mathrm{C} 20 \mathrm{~B}$ with their counterparts, $C 20 \mathrm{~B}^{\mathrm{i}}$ and $\mathrm{C} 17 \mathrm{~B}^{\mathrm{i}}$, are longer than this limit (4.640 $\AA$ ), and therefore photodimer is not expected. The same is true for the mutual relation between the two molecules $\mathrm{A}$ and $\mathrm{B}$, where one of the distances is much too long (5.191 $\AA$ ) and the overlap between the reactive p-orbitals is very poor. Moreover, dimerization of $\mathrm{A}-\mathrm{B}$ would lead to a head-to-head dimer in which the $\mathrm{N}-\mathrm{CH}_{3}$ groups will overlap, which is less probable to occur.

As expected, the photoreaction goes to completion, leading to dimers of $\mathrm{A}-\mathrm{A}$ in a single-crystal to single-crystal mode of reaction.

Three stages of the reaction are shown in Figure 3, before illumination (left), at ca. $50 \%$ conversion (middle), and after full conversion (right). There is no trace of molecules B (in green) at the intermediate stages. This implies that molecule $B$ undergoes reorientation to adopt the structure and the geometric relation of molecule $\mathrm{A}$.

Because the two molecules, A and B, are related by pseudo 2fold axis running in the plane of the pyridine ring, molecule $B$ has to flip (rotation by $180^{\circ}$ ) to have the same relative geometry of molecule A. During the flip and the photodimerization, hydrogen bonds between the light-stable and the light-sensitive molecules were broken and reformed after the dimer was produced. Further information and results will be given in the discussion. 


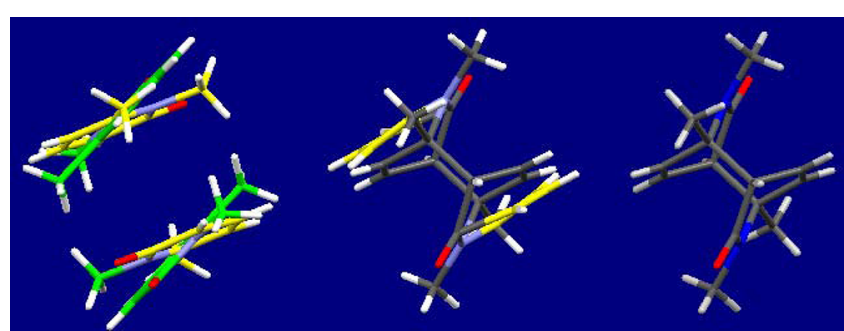

Figure 3. Structure of the light-sensitive molecules in I-a at three stages: left, before irradiation; middle, after ca. 50\% conversion; and right, at full conversion. Carbon atoms of unreacted molecule $\mathrm{A}$ are in yellow, and those of unreacted molecule B are in green.

3.2. 1,1,6,6-Tetraphenyl-2,4-hexadiyne-1,6-diol:2(2methyl-2(1H)-pyridone) (I-b). The crystal structure of I-b was published elsewhere, ${ }^{4}$ and the molecular flip was briefly mentioned. We have repeated the experiments mentioned in the previous work and will elaborate in the discussion. The guest molecule (b) is disordered with the two molecules (A and $B$ in Figure 4) related by a pseudo 2-fold axis running in the

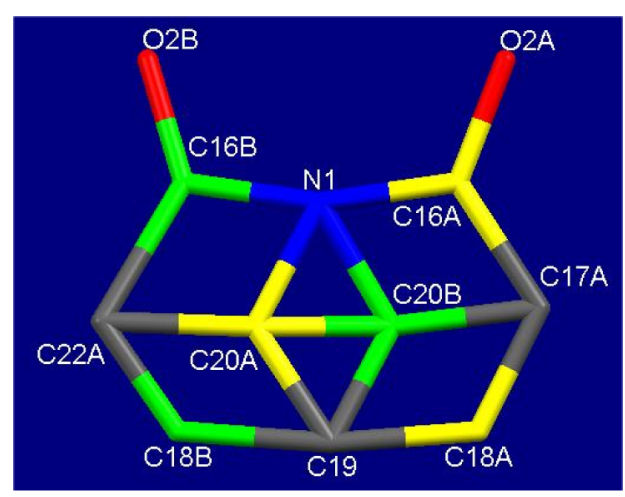

Figure 4. The disordered light-sensitive molecule in I-b.

plane of the pyridine ring, slightly different from that in I-a. The relative occupation of the two molecules A/B is $86 / 14$, respectively. The distances between the potentially reactive atoms $\mathrm{C} 17$ and $\mathrm{C} 20$ to their counterpart, $\mathrm{C} 20^{\mathrm{i}}, \mathrm{C} 17^{\mathrm{i}}$, related by inversion centers are 4.612, $3.908 \AA$ for molecules $\mathrm{A} \cdots \mathrm{A}$ and $\mathrm{B} \cdots \mathrm{B}$, respectively, 3.904, and $4.137 \AA$ (Figure 5). The arguments mentioned for the possible photodimers discussed for $\mathbf{I}-\mathbf{a}$ are valid also here. Indeed, at full conversion $\mathrm{B}-\mathrm{B}$ is the only dimer that is formed.

This is surprising, because molecules B are only 14\% from the total light-sensitive molecules in the crystal; nevertheless, they photodimerize while the majority of the molecules $(86 \%)$ have to flip (rotation be $180^{\circ}$ ) to enable the photoreaction to proceed to completion. It should be added, but not discussed,

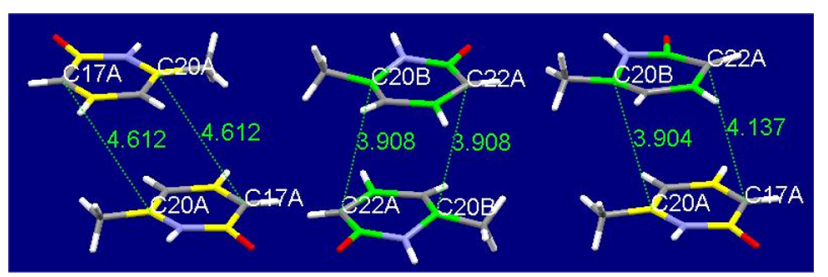

Figure 5. Relative geometry of light-sensitive molecules in I-b. A $\cdots \mathrm{A}$ (left), B $\cdots B$ (middle), and A $\cdots B$ (right). Carbon atoms of molecule A are in yellow, and those of molecule $\mathrm{B}$ are in green. that during the flip and the photodimerization, hydrogen bonds between the light-stable and the light-sensitive molecules, as well as between two neighbor light-sensitive molecules, were broken and reformed after the dimer was produced.

Further information and results will be given below in the discussion.

3.3. 1,1,6,6-Tetraphenyl-2,4-hexadiyne-1,6-diol:2(2(1H)-pyridone) (I-c). The structure of I-c was published elsewhere, ${ }^{4}$ and the flip of the light-sensitive molecules was mentioned. Therefore, we mention it here briefly for comparison with the previous two examples. The light-sensitive molecules (c) are not disordered, and they are related to each other by inversion centers. The distance between the potentially reactive atoms, $\mathrm{C} 17$ and $\mathrm{C} 20$, with their counterpart related by inversion center $\mathrm{C} 20^{\mathrm{i}}$ and $\mathrm{C} 17^{\mathrm{i}}$, is $3.835 \AA$. A priori, there is no reason for the light-sensitive molecules to flip, because the mutual geometry between pair of molecules is within any geometric limits set for photodimerization. Nevertheless, the dimers obtained show clearly that the molecules have flipped. Figure 6 shows a comparison of the structure of

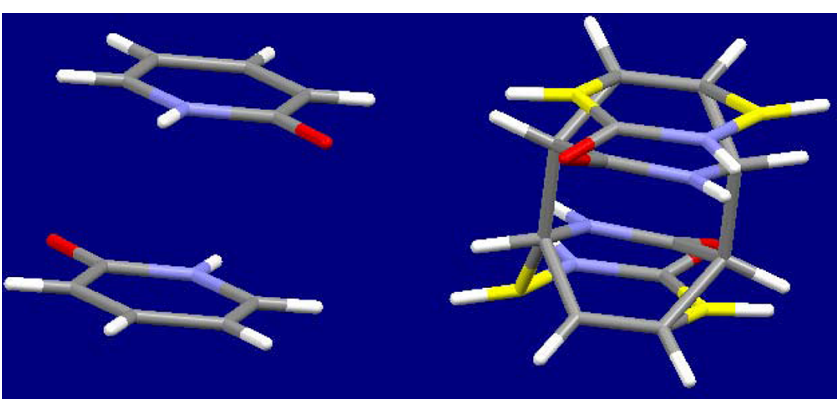

Figure 6. Structure of the light-sensitive molecules in I-b at two stages: left, before irradiation; right, after ca. $60 \%$ conversion. Carbon atoms of unreacted molecule A are in yellow, and those of unreacted molecule B are in green.

the light-sensitive molecules before irradiation (left) and when the conversion to dimers reached ca. $60 \%$ conversion (right). It is clearly seen that the remaining unreacted monomers and the dimer have a different orientation than before irradiation. This observation can be explained by flipping of the light-sensitive molecules. It should be added, but not discussed, that during the flip and the photodimerization, hydrogen bonds between the light-stable and the light-sensitive molecules, as well as between two neighbor light-sensitive molecules, were broken and reformed after the dimer was produced.

The open questions are when, why, and how do the molecules flip.

\section{DISCUSSION}

In our previous publication ${ }^{11}$ describing the crystal structure of I-b, we have suggested that the flip (or rotation) of the guest molecules belonging to the major population ( $86 \%)$ may be a result of the irradiation or of the heat evolved during irradiation. Two experiments were carried out to distinguish between the two hypotheses: first, heating the crystal without irradiation, and, second, cooling the crystal during irradiation. The temperature dependency of the two different populations of the guest molecules was estimated from the refined occupancy factors of the disordered guest molecules obtained during the crystal structure refinement process. The initial ratio between the guest populations at room temperature was 86/14 
(major/minor). It was changed to $67 / 33$ at $393 \mathrm{~K}$ and reverted to its original ratio upon cooling to room temperature. No change was observed when the crystal was cooled to $100 \mathrm{~K}$. Irradiation while cooling the crystal to $220 \mathrm{~K}$ for $20 \mathrm{~h}$ produced full conversion to the same photodimerization product as that when the photodimerization was carried out at room temperature. These results suggest that light-induced heating is not the sole cause of molecular rotation (flip). It is likely that photodimerization of molecules of the minor population shifts the equilibrium, causing molecules of the major population to flip to adopt the orientation of molecules of the minor population and thus re-establish the equilibrium, and the photodimerization proceeds until completion. We have carried out an additional experiment where the structure of the same crystal was determined at four different temperatures, and the results are shown in Figure 7. It was once again shown that the flip takes place also as a result of heat.

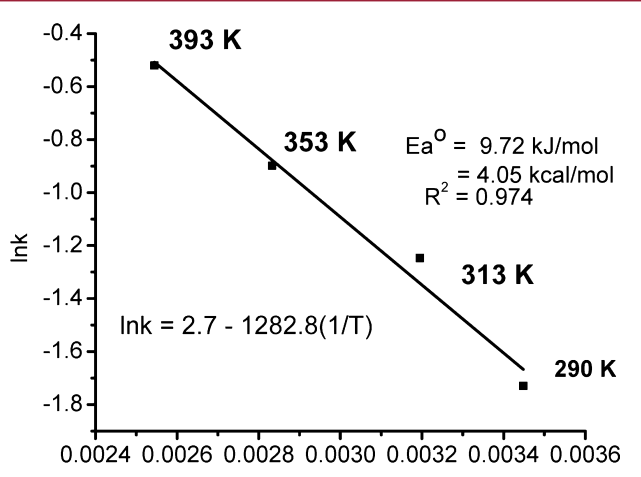

$1 / \mathrm{T}$

Figure 7. Equilibrium constants versus $1 / T$ plot for I-b. The equilibrium constant at each temperature was set to the ratio between the disordered molecules obtained by their refined occupancy factors.

The estimated activation energy of the flip is $9.72 \mathrm{~kJ} / \mathrm{mol}$. The dependence of the equilibrium between the two orientations of the light-sensitive molecules is demonstrated and justified by the effect seen in the photodimerization process, as described above.

The ratio between the disordered molecules in I-a remains constant at the range of temperatures 213-393 K. Nevertheless, the same arguments discussed for I-b hold also in this example. As soon as dimers are being formed from molecules A, the equilibrium is shifted and molecules $\mathrm{B}$ flip to adopt the orientation of molecules A. Thus, the photodimerization can proceed to completion.

In crystals of I-c, there is no disorder, and the relative geometry between light-sensitive molecules related by inversion center $\left(\mathrm{C} 17-\mathrm{C} 20^{\mathrm{i}}\right.$ distance of $3.835 \AA$ ) enables photodimerization. The photodimerization proceeds with minor changes of the unit cell dimensions; however, at certain stages of the reaction (estimated to be $\sim 50 \%$ conversion), both the remaining monomers and the formed dimers flip (see Figure 6). We have no explanation for this phenomenon.

Rotation of planar molecule such as pyridone in the plane does not need too much free space. However, rotation of such a molecule by $\sim 180^{\circ}$ perpendicular to the plane (flip) requires much space. Moreover, the molecules are arranged in pairs so that the interplanar distance is of the order of $3.5 \AA$, shorter than the length of the molecule. The rotation of the molecules B in $\mathbf{I}-\mathbf{a}$, and molecules $\mathrm{A}$ in $\mathbf{I}-\mathbf{b}$, is about an axis passing the inversion centers and running parallel to the direction $\mathrm{N} 1 \mathrm{~A} \cdots \mathrm{N} 1 \mathrm{~B}$ (in I-a) and parallel to C20A $\cdots \mathrm{C} 20 \mathrm{~B}$ (in I-b). The rotation should be concerted; the two monomers should rotate simultaneously, and this is also true for I-c.

The voids where the reactions take place were drawn using the software program Mercury (Cambridge Crystallographic Data Centre), ${ }^{17}$ which find any empty spaces (voids) that are big enough to hold spherical "probe". The radius of the probe is $1.2 \AA$, and the grid spacing is $0.7 \AA$. The voids were calculated using contact surface using the structure of the host light-stable molecules and omitting the guest light-sensitive molecules. The voids created by the host molecules and the flipping molecules are shown in Figures 8-10 for $\mathbf{I}-\mathbf{a}, \mathbf{I}-\mathbf{b}$, and I-c, respectively. The voids having a shape of slightly distorted sphere are separated from each other, and each accommodate a pair of monomers. The existing disorder in I-a and I-b suggests that there is additional space in the voids to accommodate more than that required by a single pair of molecules. The additional length available for the rotation is 1.5 and $1.4 \AA$ in $\mathbf{I}-\mathbf{a}$ and $\mathbf{I}-\mathbf{b}$, respectively (estimated from the distances $\mathrm{N} 1 \mathrm{~A} \cdots \mathrm{N} 1 \mathrm{~B}$ and C20A $\cdots$ C20B) along the rotation axis.

The fact that there is one pair of molecules in isolated cavities in each of the compounds I-a and I-b as well as the fact that the dimerization proceeds to completion suggest that there are no cavities that include the pair A $\cdots B$. The possibility that such a pair exists in a cavity is ruled out because it means that one of the molecules (A or B) would have to flip to undergo photodimerization. However, there is no space in the cavity for such a rotation. These results point out that the disorder in the

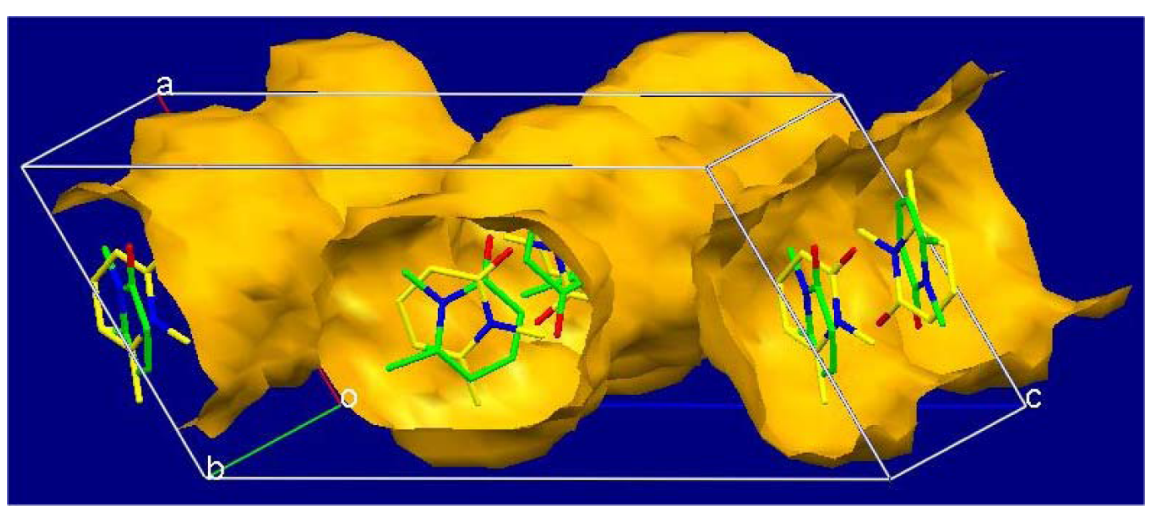

Figure 8. The reaction cavity in the crystal structure of I-a. 


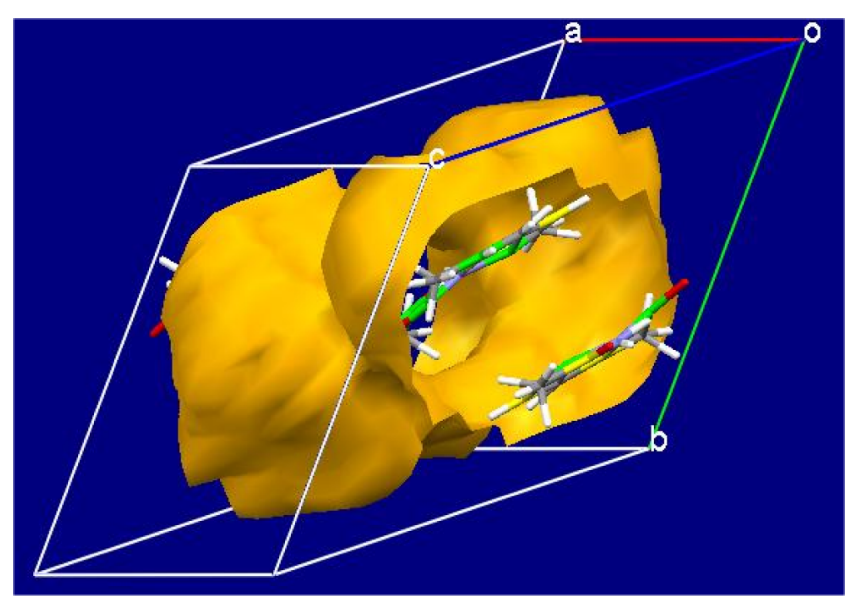

Figure 9. The reaction cavity in the crystal structure of I-b.

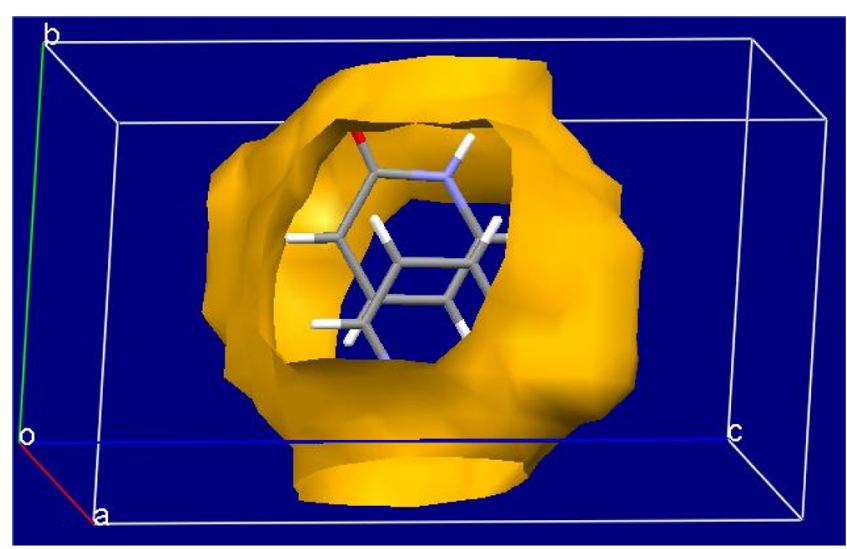

Figure 10. The reaction cavity in the crystal structure of I-c.

crystal is due to existence of cavities having either pairs of molecules of type $\mathrm{A} \cdots \mathrm{A}$ or $\mathrm{B} \cdots \mathrm{B}$. The rotation takes place, therefore, only in the cavities having the longer distances between the reactive atoms of the two molecules related by inversion center (C17 and C20) in a simultaneous fashion.

To understand the mechanism of the reaction and growth of the product, a single crystal of I-c was illuminated and checked periodically for its structure and the progress of the conversion.

Figure 11 shows the fraction of the photodimer produced (expressed in term of conversion) by the irradiation of a single crystal of I-c with $355 \mathrm{~nm}$ UV-LED. The time scale in Figure 11 is based simply on fluency of the light and is not an absolute property of the sample. The fraction of the dimer was extracted from a least-squares refinement of the crystal structure (the occupancy factor). During the refinement procedure, the sum of the occupancy factor of atoms of the monomer and the occupancy factor of the atoms of the dimer was kept to 1.0. The occupancy factor was free to refine until the residual electron density, and the $R$ value was minimal. The sigmoidal line shape of $\mathrm{JMAK}^{19-22}$ was used in the description of the $[2+2]$ photodimerization of $\alpha$-cinnamic acid. ${ }^{23,24}$ The fit of the data points was done using the equation: ${ }^{19-22}$

$$
\text { conversion }=1-\exp \left(-k t^{n}\right)
$$

where conversion $=1-$ occupancy factor.

This equation was used as a model for the kinetics of phase transition involving nucleation and growth mechanism. ${ }^{22}$ In the equation, "conversion" is the fraction of the dimer, $t$ is the

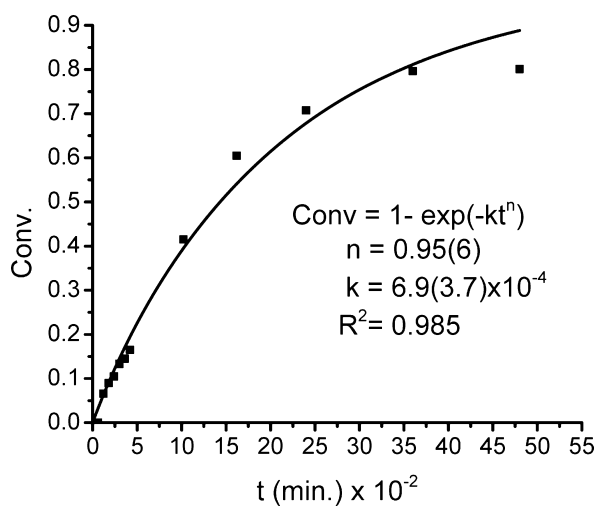

Figure 11. Plot of the fraction of the light-sensitive molecule of I-c as a function of the irradiation time at room temperature. The data points (घ) are the refined occupancy factors, and the line is a fit using the equation and the parameter shown. The time scale is based simply on fluence of the Xe light and is not an absolute property of the sample.

exposure time, $k$ is the constant of the growth rate, and $n$ is the Avrami exponent that shows the dimension of the growth. When $n=2,3$, or 4 , the dimensionality of the growth is 1,2 , and 3 , respectively. When $n=1$, it means that the nucleation rate is constant over time and that the reaction is homogeneous with equal probability to occur in any region of the sample. In a plot of $\ln \left[\ln (1-y)^{-1}\right]$ versus $\ln$ [time], called an "Avrami plot", the parameters of the reaction can be easily extracted. ${ }^{2,23} \mathrm{~A}$ straight line with slope $n$ is obtained. Figure 12 shows the

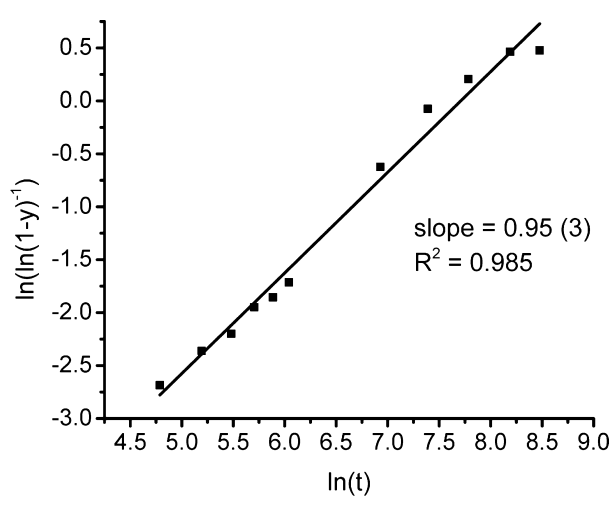

Figure 12. Avrami plot of $\ln \left[\ln (1-y)^{-1}\right]$ versus $\ln ($ time $)$, where $y$ is the fraction of the conversion. The slop is the Avrami exponent.

Avrami plot for the experimental data. The linearity of the plot indicates that this is a JMAK kinetic process. The slope of 0.95 (3) is identical to that obtained above. Bertmer et al. have used the Avrami model to fit the sigmoidal curve observed in the kinetic study of the $[2+2]$ photodimerization of $\alpha$-transcinnamic acid. ${ }^{23}$ The Avrami exponent was found to be 1.66(10); Benedict and Coppens ${ }^{24}$ have found 1.43(8) for the same reaction using a two-photon absorption experiment. Avrami exponents of 1.6(1) and 1.5(1) were found for the [4+ 4] photodimerization of 1,4-dimethyl-2-pyridone in its cocrystal with 1,1,6,6-tetraphenyl-2,4-hexadiyne-1,6-diol at 230 and $280 \mathrm{~K}$, respectively. ${ }^{10}$ It was found that different substituents at the aromatic ring of the cinnamic acid influence the reaction mechanism. For example, it was found ${ }^{25}$ that the Avrami exponent is $0.98(11)$ for the kinetics curve of $o$ methoxy cinnamic acid, and it was $2.22(11)$ for the kinetic curve of $o$-ethoxy cinnamic acid. 
The first is indicative of a heterogeneous one-dimensional linear growth, and the second is indicative of heterogeneous two-dimensional growth, with a decreasing nucleation rate over time. The chemical system in the present work consists of lightsensitive guest molecules that are almost isolated within cavities formed by light-stable host molecules; therefore, we expected different behavior. We have found that $n=0.95(6)$, indicating that the growth mechanism is similar to the kinetics of $o$ methoxy cinnamic acid. ${ }^{25}$ A very different Avrami exponent of $0.55(8)$ was recently reported for the $[4+4]$ photodimerization of 9-anthracene-carboxylic acid. ${ }^{26}$ An Avrami exponent of 0.55 would lead to a negative dimensionality, which might suggest an auto inhibition step in the reaction. It is important to note that in this particular case the two monomers are related by crystallographic translation leading to head-tohead orientation ( $\beta$-type packing); therefore, the carboxylic acid groups have to adjust their conformations to enable dimerization with minimal interference between them. This might lead to additional disorder in the crystal, as suggested by the authors.

\section{CONCLUSION}

It was shown that in illumination by UV light of three examples of molecular compounds composed of light-stable host and light-sensitive guest molecules, $[4+4]$ photodimerization takes place. In these examples, the dimerization takes place with molecular flip. The photochemical reaction as well as the molecular flip could be followed due to the fact that the whole process proceeds in a single-crystal to single-crystal type transformation. The driving force for the molecular flip is well understood in the two compounds where the light-sensitive molecules are disordered (I-a and I-b). It seems that the driving force for the molecular flip in I-c is free-energy minimization if the space provided for the flip is available.

It was shown that the molecular flip in I-b is an equilibrium controlled process with an estimated activation energy of 9.72 $\mathrm{kJ} / \mathrm{mol}$. The kinetics of the reaction and crystal growth mechanism was studied for compound I-c, which indicate that the nucleation rate is constant over time and that the reaction is homogeneous with equal probability to occur in any region of the sample.

\section{ASSOCIATED CONTENT}

\section{S Supporting Information}

X-ray crystallographic information files (CIF) for compounds Ia, I-b, and I-c, tables of crystal data and refinement (S1, S2, and S3), and crystal structures before and after irradiation. This material is available free of charge via the Internet at http:// pubs.acs.org.

\section{AUTHOR INFORMATION}

\section{Corresponding Author}

*E-mail: kaftory@tx.technion.ac.il.

\section{Present Addresses}

${ }^{\ddagger}$ State Key Laboratory of Coordination Chemistry, Coordination Chemistry Institute, Nanjing University, Nanjing 210093, People's Republic of China.

${ }^{\S}$ Structural Dynamics of (Bio)Chemical Systems, Max-PlanckInstitute for Biophysical Chemistry, Am Fassberg 11, 37077 Göttingen, Germany.

\section{Notes}

The authors declare no competing financial interest.

\section{ACKNOWLEDGMENTS}

This work was supported by the Israel Science Foundation, no. 499/08.

\section{REFERENCES}

(1) Lavy, T.; Sheynin, Y.; Sparkes, H. A.; Howard, J. A. K.; Kaftory, M. CrystEngComm 2008, 10, 734.

(2) Lavy, T.; Sheinin, Y.; Kaftory, M. Eur. J. Org. Chem. 2004, 48024808.

(3) Zouev, I.; Lavy, T.; Kaftory, M. Eur. J. Org. Chem. 2006, 41644169.

(4) Lavy, T.; Kaftory, M. CrystEngComm 2007, 9, 123-127.

(5) (a) Amirsakis, D. G.; Elizarov, A. M.; Garcia-Garibay, M. A.; Glink, P. T.; Stoddart, J. F.; White, A. J. P.; William, D. J. Angew. Chem., Int. Ed. 2003, 42, 1126-1132. (b) Amirsakis, D. G.; GarciaGaribay, M. A.; Rowan, S. J.; Stoddart, J. F.; White, A. J. P.; Williams, D. J. Angew. Chem., Int. Ed. 2001, 40, 4256-4261 and references therein.

(6) Toda, F.; Bishop, B. Separation and Reactions in Organic Supramolecular Chemistry: Perspectives in Supramolecular Chemistry; John Wiley and Sons: New York, 2004; Vol. 8.

(7) Ananchenko, G. S.; Udachin, K. A.; Ripmeester, J. A.; Perrier, T.; Coleman, A. W. Chem.-Eur. J. 2006, 12, 2441-2447.

(8) Halder, G.; Kepert, C. Aust. J. Chem. 2006, 59, 597-604.

(9) Coppens, P.; Zheng, S.-L.; Gembicky, M.; Messerschmidt, M.; Dominiak, P. M. CrystEngComm 2006, 8, 735-741.

(10) Cao, D.-K.; Sreevidya, T. V.; Botoshansky, M.; Golden, G.; Benedict, J. B.; Kaftory, M. J. Phys. Chem. A 2010, 114, 7377-7381.

(11) (a) Chong, K. C. W.; Sivaguru, J.; Shichi, T.; Yoshimi, Y.; Ramamurthy, V.; Scheffer, J. R. J. Am. Chem. Soc. 2002, 124, 28582859. (b) Joy, A.; Uppili, S.; Netherton, M. R; Scheffer, J. R; Ramamurthy, V. J. Am. Chem. Soc. 2000, 122, 728-729. (c) Leibovitch, M.; Olovsson, G.; Sundarababu, G.; Ramamurthy, V.; Scheffer, J. R.; Trotter, J. J. Am. Chem. Soc. 1996, 118, 1219-1220.

(12) (a) Adams, R.; Schreker, A. W. J. Am. Chem. Soc. 1949, 71, 1186. (b) Cook, D. J.; Boen, R. E.; Sorter, P.; Daniels, E. J. Org. Chem. 1961, 26, 4949.

(13) Kuwatani, Y.; Yamamoto, G.; Oda, M.; Iyoda, M. Bull. Chem. Soc. Jpn. 2005, 78, 2188-2208.

(14) Nonius. COLLECT; Nonius BV: Delft, The Netherlands, 2000.

(15) Otwinowski, Z.; Minor, W. In Methods in Enzymology, 276, Macromolecular Crystallography, Part A; Carter, C. W., Jr., Sweet, R. M., Eds.; Academic Press: New York, 1997; pp 307-326.

(16) Sheldrick, G. M. SHELXS97 and SHELXL97; University of Göttingen: Germany, 1997.

(17) Macrae, C. F.; Edgington, P. R.; McCabe, P.; Pidcock, E.; Shields, G. P.; Taylor, R; Towler, M.; van de Streek, J. J. Appl. Crystallogr. 2006, 39, 453-457.

(18) (a) Schmidt, G. M. J. J. Chem. Soc. 1964, 2014. (b) Schmidt, G. M. J. Pure Appl. Chem. 1971, 27, 647.

(19) Avrami, M. J. Chem. Phys. 1939, 7, 1103-1112.

(20) Avrami, M. J. Chem. Phys. 1940, 8, 212-224.

(21) Avrami, M. J. Chem. Phys. 1941, 9, 177-184.

(22) Christian, J. W. The Theory of Transformations in Metals and Alloys, Part I; Elsevier Science Ltd.: Oxford, UK, 2002; Vol. 1.

(23) Bertmer, M.; Nieuwendaal, R. C.; Barnes, A. B.; Hayes, S. E. J. Phys. Chem. B 2006, 110, 6270-6273.

(24) Benedict, J.; Coppens, P. J. Phys. Chem. A 2009, 113, 31163120.

(25) Fonseca, I.; Hayes, S. E.; Bertmer, M. Phys. Chem. Chem. Phys. 2009, 11, 10211-10218.

(26) More, R.; Busse, G.; Hallmann, J.; Paulmann, C.; Scholz, M.; Techert, S. J. Phys. Chem. C 2010, 114, 4142-4148. 